\title{
Microbiology: the return of culture
}

\author{
Vivien Marx
}

Sequencing technology drives microbiology and gives researchers new reasons to draw on classic techniques.

The number might be $100,1,000$ or 1,500 . Three scientists might offer three different numbers when asked how many bacterial species a healthy gut contains, says Trevor Lawley, a microbiologist at the Wellcome Trust Sanger Institute. Sequencing approaches have yet to reveal definitive answers.

Sequencing, notably 16 S ribosomal RNA (rRNA) gene amplification and sequencing, and metagenomics, in which genetic material from samples chock full of microbial species are sequenced in one go, has galvanized microbiology. As microbiome research has grown, sequencing has become a standard assay to identify and count microbes and their genes. Ideally, sequencing provides a complete census of a sample, but often the census data have gaping holes. These methods generate data about genome fragments, not entire genomes, says Lawley. There are too few reference genomes with which to assign these data to a known species, and many species remain unidentified because they do not grow in the lab. The microbiome field has not focused on its foundation, which is microbiology, says Lawley. "It's a major deficiency."

For years, researchers have disregarded the use of culture to establish the repertoires of microbes associated with the gut,

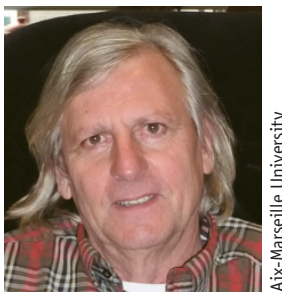

Metagenomics is powerful, says Didier Raoult, but it needs to be complemented with 'culturomics'.

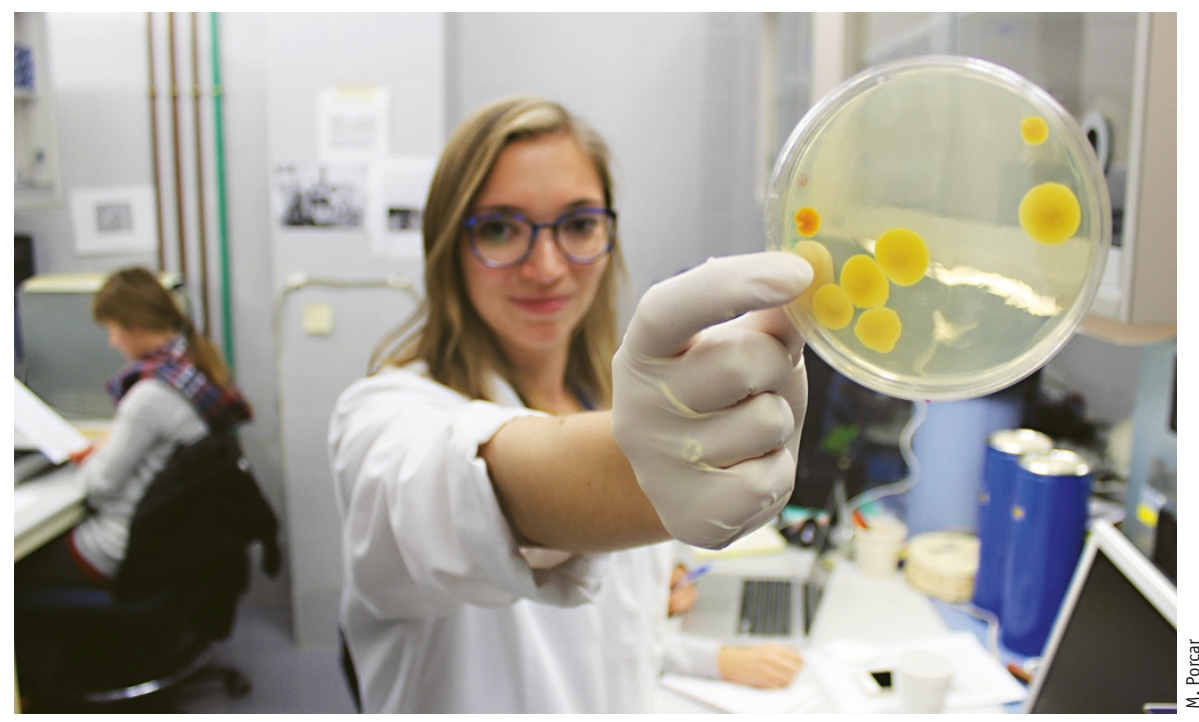

A culture revival is underway. Culture is being combined with methods such as omics, phenotyping, and mass spectrometry.

says Didier Raoult,

key to a newborn's immune system or coral reef health, have pathogenic traits, or might be healthful in a probiotic drink.

The good news is that many researchers have realized what they need to do to fill the gaps in data. "We are living in a revival of the culture," says Raoult. "Culturing is going to live a 'second youth', in parallel with the massive use of 'omics"' This revival combines culture with other methods, such as phenotyping, mass spectrometry, in situ hybridization and omics. at Aix-Marseille University. Culture is the classic task of coaxing microbes to grow on agar plates. Labs want to identify and grow microbes to explore how they function and discover, for example, which bacterial species are

\section{Vive la culture}

Metagenomics is unquestionably powerful, says Raoult, but it needs to be complemented by what he calls 'culturomics', which combines culturing, sequencing and mass spectrometry to identify bacteria in samples ${ }^{1}$. Metagenomics results are hard to compare between labs-they may use differing DNA extraction methods, for example. Escherichia coli produces polysaccharides that are potent PCR inhibitors. A remedy is the mechanical lysis of samples plus degradation of these polysaccharides. But not every research team will have done this, which is why he advises caution for data comparison.

Often a bug is difficult to grow in the lab. Raoult has long focused on growing difficult bacteria, including many species of the pathogen Rickettsia. He and his team also used PCR to identify Yersinia pestis-specific sequences in dental pulp extracted from skeletons in mass graves dating back to the sixteenth and eighteenth centuries. Over time, he expanded his approaches, combining culturing, 16S rRNA sequencing and matrix-assisted laser desorption/ionization-time of flight (MALDI-TOF) to create a high-throughput way to analyze samples to find what might otherwise be missed.

Raoult says he likes the speed of MALDITOF and its role as a discovery tool. Species have unique protein signatures that 
correspond to different mass spectra. He has eight MALDI-TOF instruments in his lab. Each costs $\$ 100,000$ to $\$ 150,000$, and the cost of reagents is negligible; "this is why we can test so much," he says. His lab has generated a total of 10 million mass spectra from between 3 million and 5 million microbial colonies tested. The team puts the spectra in the database that comes with the Bruker instruments, and the lab also has a homebuilt database.

Raoult and his group also worked out 212 distinct medium-based culture conditions, such as preincubation in a blood culture bottle or the addition of sheep blood or rumen fluid, which is not commercially available and must be prepared in the lab. He and his team have reduced the list to 18 and are currently whittling it down to 5 , he says.

Applying culturomics, Raoult and his team recently analyzed nearly 1,000 stool samples and 30 samples from the small intestine and colon of people in Europe, rural and urban Africa, Polynesia and India. They tested nearly 1 million colonies and discovered previously uncultured microbes from the human gut ${ }^{2}$. They identified more than 1,525 bacterial species in the fecal samples, including more than 140 human bacteria not previously known to be in the gut, more than 180 bacteria and 1 archaeal species not previously isolated from humans, and almost 200 previously unknown bacterial species.

The scientists used magnifying glasses to detect tiny 'microcolonies', some as small as $100 \mu \mathrm{m}$ in diameter, on culture plates. They grew these colonies and performed MALDI-TOF or $16 \mathrm{~S}$ rRNA analysis. To culture methanogenic bacteria, which are expensive and highly challenging, they used a double-chambered assay in which
Bacteroides thetaiotaomicron grows in the lower chamber, producing hydrogen that helps the methanogenic bacteria in the upper chamber to grow.

Culturomics and metagenomics stand side by side, says Raoult; each shows scientists a different part of the microbial world. "It's not inferior, it's not superior, it's a different part of the world," he says. Culturomics helps with finding and culturing bacterial species. Labs can then use these discoveries to help annotate or re-annotate metagenomic data, creating a microbiological El Dorado of sorts, he says. Over the past 2 years, a PhD student in Raoult's lab has discovered 50 bacterial species. Many of the lab's 18 members discover 3-10 new microbial species a week.

Discovery matters for science and potential applications (see Box 1). When microbiologist Ruth Ley, director of the

\section{BOX 1 MICROBIOLOGY-BASED PROSPECTING}

Manuel Porcar and Cristina Vilanova, of the University of Valencia, and their colleagues are self-professed 'bioprospectors.' They hunt for organisms, especially microbes that have potential for many applications. Porcar cofounded the company Darwin Bioprospecting Excellence in early 2016. Vilanova joined later that year after completing her $\mathrm{PhD}$. The company offers bioprospecting services for academic labs in Europe and North America as well as for small and mediumsized companies in the biotech and agro-food industry, says Vilanova. The team combines sequencing, culturing and other techniques. They are also characterizing strains they have collected to explore their potential in areas such as bioremediation, biomass conversion and food processing. "There's always a bunch of microbes naturally trained to do whatever you need," says Vilanova.

In both their lab and their company, the researchers bioprospect with a view to industrial applications. Using omics techniques and culturing, they have, for example, found a diversity of microorganisms on solar panels. And the diversity increases the chances of finding a microbe they can isolate and 'domesticate' in the lab through culturing to test these microbes' potential, says Vilanova. For example, they hunt for "super-producers" of carotinoids, which are used in the pharmaceutical and cosmetics industries.

Although microbial genes or pathways can be chosen and cloned into E. coli or yeast, Porcar says the team is focusing on culturable organisms from the environment as potential biofactories to make metabolites of interest. These microbes promise to be more robust for many applications, and their use avoids regulatory issues related to genetically modified organisms.

The estimated 1 trillion bacterial species on Earth represent huge biodiversity, says Porcar. Even if the estimate is off, there are also subspecies and strains to consider. A metagenomics-centered comprehension of the biosphere and

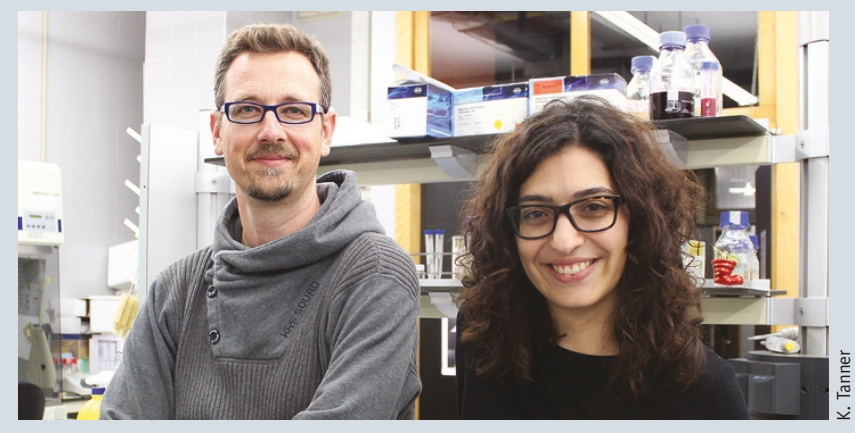

Manuel Porcar and Cristina Vilanova hunt for microbes they can 'domesticate' in the lab.

the fragmentary state of much metagenomic data will not suffice for discovering this biodiversity. Annotation methods and databases are improving, says Vilanova, but labs cannot currently identify the total diversity of microorganisms in their environmental samples. And the complex data sets still have to be analyzed for the core information they hold, especially for applied research.

Many extreme and unexplored habitats are likely to harbor novel species or gene sequences, but omics techniques alone will not be enough to find them. The genome is "just the shadow of an organism; it's not it," says Porcar. "If you can culture a microorganism, it's literally on your hands." Metagenomics can help to discover industrial enzymes, for example, but strain-level information is hard to obtain, especially when the microbes cannot be cultured. That is why, he says, "culture-dependent and -independent techniques need each other." Many microbiome-focused companies have emerged, and in this competitive environment, the details of Darwin's approach are proprietary. What differentiates the approach from others, he says, is a focus on improving culturing methods to "isolate the unisolatable." 


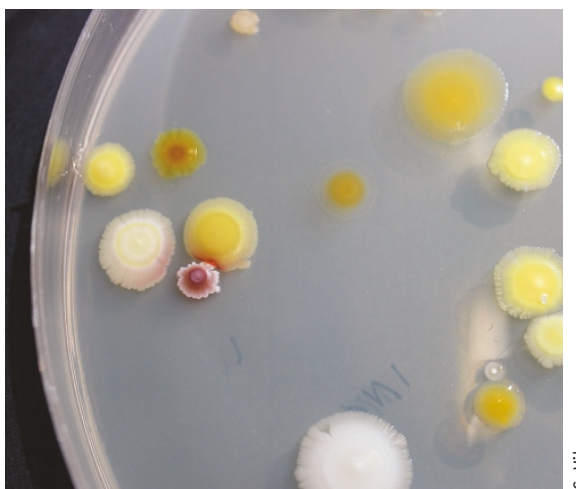

Culture methods coax microbes to grow on agar plates, enabling study of their functions.

Max Planck Institute for Developmental Biology, was at Cornell University, she and her colleagues isolated a new anaerobic bacterium from the gut, Christensenella minuta. Ley and her team showed weight loss and less adiposity in mice with this bacterium in their gut. And some researchers think C. minuta could become part of a probiotic with which to study and possibly treat obesity. Raoult's lab uncovered two more Christensenella species, C. massiliensis and C. timonensis. These microbial species are difficult to isolate and grow.

A gene sequence can indicate a physiological role, as in the case with C. minuta, says Raoult, but to establish the scientific basis for a microbe's metabolic effect, labs need to grow, sequence and characterize it. His group is discovering too many species to be able to characterize all of their biology, he says. But others can use the spectra, genomic data and strains. Ultimately, he says, labs need the bug. And they need microbiologists.

Some microbiome researchers hail from computational biology, and others focus on phylogeny, but microbiologists have a valuable biological perspective for researching the gut, says Raoult. For example, many gut disorders are connected to anaerobic bacteria, so labs must tend to the differences between aerobes and anaerobes. "Microbiota need microbiologists," he says.

At the Sanger Institute, Lawley and his team considered mass spectrometry-based methods, and he agrees that they are fast and inexpensive. Mass spectrometry also offers "a shorter-term gain with identification," he says. But a mass spec signature from a previously uncultured microbe means that labs still have to sequence its genome, so Lawley prefers sequencing over mass spectrometry to accompany culturing methods. "I'm at a genome center; that's what we do," he says.
But sequencing is not the first step in his workflow, nicknamed CRAP, for 'culture, "We flipped it around," says Lawley of the approach. He starts with microbial phenotype, applies large-scale culturing, and then follows with sequencing.

\section{Start with phenotype}

To test this workflow, Lawley and his team studied a distinct, metabolically dormant state of bacterial life: sporulation ${ }^{3}$. Spores are a cellular phenotype of a bacterial species or strain; a thick shell envelops the bacterium and it is resistant to oxygen and ethanol.

The researchers did targeted phenotyping by testing for ethanol-resistant spores in the human gut microbiome. They isolated 137 bacterial species, 45 of which were potentially novel. They also cultured 90 species from the Human Microbiome Project's 'mostwanted list' of uncultured and unsequenced microbes.

The team plated the spores, coaxing them to germinate and return to active growth using YCFA agar, to which glucose, maltose and cellobiose were added. The plates were subjected to anaerobic conditions, and intestinal bile salts were added. Next came sequencing to find the genotype linked to the sporulation phenotype.

Lawley and his team call sporulation an "unappreciated" phenotype that might, for example, help researchers to better understand bacterial transmission. The human gut contains many anaerobic bacteria that re-streaking, archiving and phenotyping.

are transmitted between individuals, such as Clostridium difficile, which causes diarrhea. Somehow this anaerobic bacterium survives exposure to air, a feat that is perhaps enabled by sporulation.

The researchers found spore-forming bacteria in the microbiota to be much more diverse than non-spore-forming bacteria. Exploring spores will help to annotate metagenomic data that indicate that the gut

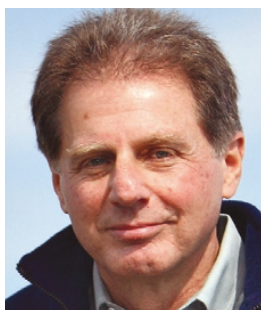

Gary Borisy is scaling up FISH to explore microbial communities.

\section{tional differences between the microbiomes} of individuals, for example.

Sequencing is not a 'functional tell-all'. For example, says Lawley, microbes might have a gene known to confer resistance to a particular antibiotic. But the gene's presence does not mean the organism is resistant, he says. Combining metagenomics with phenotyping and culturing helps to discern which microbes are present and offers functional insight. When scientists use only metagenomic approaches, they make

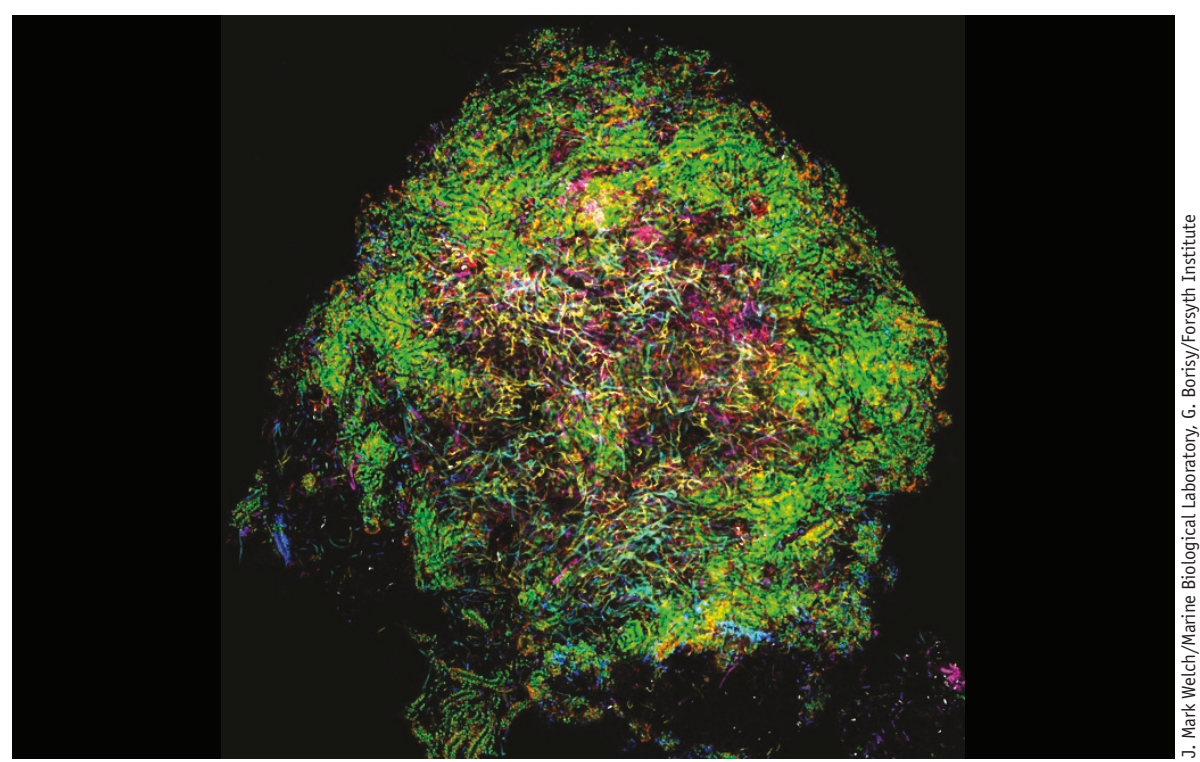

The spatial organization of microbial community in human dental plaque, rendered visible at the micrometer scale by FISH. 


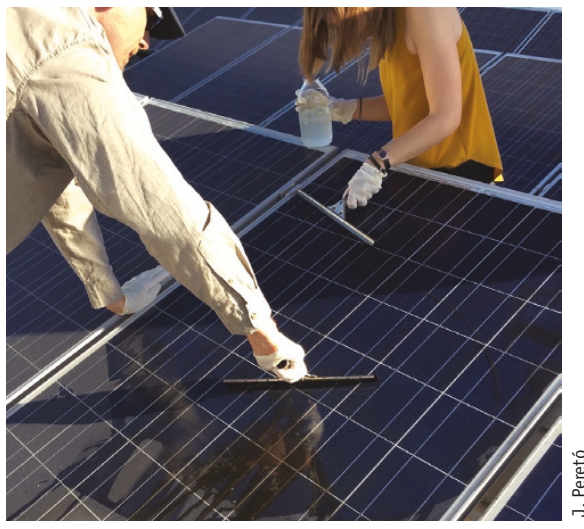

Using omics techniques and culture, researchers at the University of Valencia and the startup Darwin Bioprospecting Excellence have found diverse microorganisms on solar panels.

assumptions about phenotypes. Yet they still must do experiments to see whether an assumption is true, he says, if they want, for example, to understand disease-causing anaerobes in the human gut.

Conceptually, says Lawley, his approach addresses the 'great plate count anomaly', a longstanding frustration in microbiology in which many microbes can be detected and phenotyped but not grown in the lab for further analysis. In his view, the CRAP workflow avoids this situation by rendering many more, and perhaps all, microbes culturable and opens up the human microbiome for phenotypic and functional analysis. "We can culture almost everything," he says. "The dogma of the unculturable doesn't really exist for the human microbiome." It has been replaced by trial and error to find the ideal growth media, nutrients and conditions microbes need.

\section{In situ approaches}

At the California Institute of Technology, Victoria Orphan and her team characterize microbiomes in extreme environments, such as methane-producing bacteria in deep-sea locations. She applies a combination of molecular biology techniques, mass spectrometry and methods such as fluorescence in situ hybridization (FISH) to functionally analyze the microbes. For example, she and her team use bio-orthogonal noncanonical amino acid tagging (BONCAT-FISH) to label proteins that a microbe is actively producing to assess metabolic activity ${ }^{4}$.

Another emerging in situ method uses fluorescence to capture the spatial qualities of a microbiome, such as in oral biofilms. Gary Borisy at the Forsyth Institute and Jessica Mark Welch at the Marine Biological
Laboratory have adapted FISH to target rRNA and identify a wealth of microbes distinctly, in a single experiment ${ }^{5}$. Their approach is called combinatorial labeling and spectral imaging (CLASI)-FISH.

Metagenomics is powerful, says Borisy, but its weakness is that the experiment begins with grinding a sample to extract DNA. "In the process, you lose all spatial information at micron-to-millimeter scales," he says. The dynamics of individual cells in the sample are also lost. Metagenomics offers data about the genetic potential of a community, but "it doesn't say whether that potential is expressed." That requires meta-transcriptomics and genome assembly, which are challenging. Beyond knowing about genes and gene expression, scientists want to connect this information to the molecular physiology of individual taxa and assess how microbes interact in a community.

On its own, says Borisy, metagenomics cannot address all of a microbiome's dimensions, and this realization led him to explore scaling up FISH. "It can't be scaled up like DNA sequencing, but we think it can be pushed further," he says. He and his team have begun using binary labels as a practical way to obtain a large number of fluorescent signatures. The team did a "proof-of-imaging test" with E. coli and showed that with 16 individual fluors they could distinguish 120 binary combinations of labels. In principle, he says, it's possible to use higher-order combinations and expand the number of label types. Interpreting the resulting emission spectra involves linear unmixing, and the linear equations to do this are usually built into platforms such as MATLAB. "We use in-house scripts drawing upon MATLAB functions," he says. Most manufacturers of spectral imaging systems build unmixing operations into their software.

Borisy and his team are now working on how to address variable brightness and signal strength of CLASI-FISH fluorophores. Probes also differ in hybridization efficiency, he says, and it can be a challenge to get the probes across the bacterial envelope. Ultimately, he says, the method can deliver species-level information because probes can be designed at almost any phylogenetic level. "Of course, probe design becomes more difficult when one is trying to differentiate closely related taxa that might differ in only one or two bases," he says. For probe design, he draws on sequence databases, hybridization know-how and tools such as the web-based probe design tool mathFISH.
Each computer-designed probe still has to be validated for efficacy and specificity in experiments run with a range of taxa.

Borisy and his colleagues have used CLASI-FISH to map microbes at the genus level, and they reached species level for a few of the genera. "We are pushing harder in this direction now," says Borisy. They collected 3D data, which is important because biofilms are not monolayers but 3D communities. CLASI-FISH lets scientists "look more holistically" at a microbial community than is possible with one or a few probes, he says. In this sense, the method helps labs understand complete communities as well as their range of variability, which he hopes will make it easier to compare and contrast results from different labs.

In Lawley's view, a microbiome's spatial organization is "pretty key." He has seen CLASI-FISH data and likes the patterns it reveals. Lawley sees his approach as complementary to phenotyping accomplished with CLASI-FISH. Once bugs are cultured, labs can use antibodies and other reagents, as well as FISH-based approaches, to explore how species interact in a microbial community.

An estimated $10^{11}$ to $10^{12}$ microbial species inhabit Earth, according to Indiana University researchers Jay Lennon and Kenneth Locey ${ }^{6}$. Of these microbes, around $10^{4}$ species have been cultured and fewer than $10^{5}$ are represented in annotated genome sequence information. A number of microbiologists believe that characterizing these riches will require both cultureindependent sequencing and classic microbiology techniques ${ }^{2,3,7}$. This combination has potential for applications, too. Together, the methods might let microbiologists bid adieu to the great plate count anomaly and turn uncultivable microbes into ones that can be studied in the lab.

\section{Vivien Marx is technology editor for}

Nature Methods (v.marx@us.nature.com).

1. Lagier, J.-C. et al. Clin. Microbiol. Rev. 28, 237-264 (2015).

2. Lagier, J.-C. et al. Nat. Microbiol. 1, 16203 (2016).

3. Browne, H.P. et al. Nature 533, 543-546 (2016).

4. Hatzenpichler, R. et al. Environ. Microbiol. 16 2568-2590 (2014).

5. Mark Welch, J.L. et al. Proc. Natl. Acad. Sci. USA 113, E791-E800 (2016).

6. Locey, K.J. \& Lennon, J.T. Proc. Natl. Acad. Sci. USA 113, 5970-5975 (2016).

7. Vilanova, C. \& Porcar, M. Nat. Microbiol. 1, 16101 (2016). 